\title{
Correction to: Two Magnetic Sensor Based Real-Time Tracking of Magnetically Inflate Swallowable Intragastric Balloon
}

\author{
Muneaki Miyasaka (10, ${ }^{1}$ Anthony Meng Huat Tiong, ${ }^{1}$ Phuoc Thien Phan, ${ }^{1}$ \\ Yanpei Huang, ${ }^{1}$ Hung Leng KaAn, ${ }^{2}$ Khek Yu Ho, ${ }^{3}$ and Soo Jay Phee ${ }^{1}$ \\ ${ }^{1}$ School of Mechanical and Aerospace Engineering, Nanyang Technological University, Singapore, Singapore; ${ }^{2}$ Department of \\ General Surgery, National University Hospital, Singapore, Singapore; and ${ }^{3}$ Department of Medicine, National University of \\ Singapore, Singapore, Singapore

\section{Correction to:} \\ Annals of Biomedical Engineering (2021) \\ https://doi.org/10.1007/s10439-020-02716 \\ This Erratum is to correct equations 6, 7, 8, and 9. \\ $R_{I z}=\left[z_{\mathrm{top}}, z_{\mathrm{top}}+v_{\max } \Delta t\right] \in\left[z_{\mathrm{top}}, z_{0}\right]$ \\ $R_{I p}=\left[0, \theta_{\mathrm{p}, \max }\right]$

$$
\begin{aligned}
& \boldsymbol{B}_{\text {thresh }_{j}, \boldsymbol{k}+\boldsymbol{I}}=\boldsymbol{B}_{\text {thresh }_{j}, \boldsymbol{k}}+\boldsymbol{K}_{\boldsymbol{p}_{j}, \boldsymbol{k}}(z) e_{j, k}+\boldsymbol{K}_{\boldsymbol{d}_{j}, \boldsymbol{k}} \frac{d e_{j, k}}{d t} \\
& \boldsymbol{K}_{\boldsymbol{p}_{j}, \boldsymbol{k}}(z)=\alpha(z)\left|\boldsymbol{B}_{\text {sensor }_{j}, \boldsymbol{k}}\right| \\
& \boldsymbol{K}_{\boldsymbol{d}_{j}, \boldsymbol{k}}=\beta\left|\boldsymbol{B}_{\text {sensor }_{j}, \boldsymbol{k}}\right| \\
& e_{j, k}=n_{\text {target }}-n_{j, k} \\
& \frac{d e_{j, k}}{d t}=\frac{e_{j, k}-e_{j, k-1}}{t_{k}-t_{k-1}}
\end{aligned}
$$

$R_{I z}=\left[z_{k}-z_{u n c}, z_{k}+v_{\max } \Delta t+z_{u n c}\right] \in\left[z_{0}, z_{E G J}-d\right)$

$R_{I p}=\left[\theta_{p, k}-\phi_{R 1}-\theta_{p, u n c}, \theta_{\mathrm{p}, k}+\phi_{R 1}+\theta_{p, u n c}\right] \in\left[0, \theta_{p, \max }\right]$

$R_{I z}=\left[z_{k}-z_{\text {unc }}, z_{k}+\dot{z}_{k} \Delta t+\frac{1}{2} g \Delta t^{2}+z_{\text {unc }}\right] \in\left[z_{E G J}-d, z_{\text {bottom }}\right]$

$R_{I p}=\left[\theta_{\mathrm{p}, k}-\phi_{R 2}-\theta_{p, \text { unc }}, \theta_{p, k}+\phi_{R 2}+\theta_{p, \text { unc }}\right] \in[0, \pi]$
The original article has been corrected.

Publisher's Note Springer Nature remains neutral with regard to jurisdictional claims in published maps and institutional affiliations.

Address correspondence to Muneaki Miyasaka, School of Mechanical and Aerospace Engineering, Nanyang Technological University, Singapore, Singapore. Electronic mail: mmiyasaka@ntu. edu.sg

The original article can be found online at https://doi.org/10 1007/s10439-020-02716. 